\title{
Creencias sobre el juego: construcciones desde el contexto y la experiencia ${ }^{1}$
}

\author{
Beliefs about the game: constructions \\ from context and experience
}

Diana Alexandra Ariza Vanegas ${ }^{2}$ Johanna Marcela Sánchez Buitrago ${ }^{3}$

\section{Resumen}

Este artículo es el resultado del proyecto de investigación: Creencias sobre el juego de docentes de los programas de formación de maestros para la educación inicial de Bogotá de la Licenciatura en Educación Preescolar de la Fundación Universitaria Monserrate - Unimonserrate y de la Licenciatura en Pedagogía Infantil de la Universidad Javeriana. Se realizó desde un enfoque cualitativo y desde una perspectiva hermenéutica, a través de la entrevista semiestructurada y del análisis del documento institucional Plan de Estudios. Se fijó como propósito comprender las creencias sobre el juego en el ámbito universitario y propiciar un acercamiento a este objeto de estudio, como una posibilidad didáctica que amerita ser explorada por los futuros maestros.

Uno de los principales hallazgos hace referencia a la relación de las creencias de los docentes con sus experiencias en la etapa infantil, su formación profesional y el contexto donde laboran.

Palabras clave: Creencias; Juego; Maestros; Experiencia y contexto.
This article presents the research project: Beliefs about the teacher's game of teachers in training programs for the initial education in Bogotá of the Bachelor's Degree in Early Childhood Education from Fundacion Universitaria Unimonserrate and the Bachelor's Degree in Child Pedagogy from Universidad Javeriana, which was carried out from a qualitative and from a hermeneutic perspective, through the semi-structured interview and the analysis of the institutional document Course Plan.

As a purpose it was set to understand the beliefs about game in the university environment and to promote an approach to this object of study, as a didactic possibility that must be explored by future teachers.

One of the main findings refers to the relationship of teachers' beliefs with their experiences in the childhood stage, their professional training and the context in which they work.

Keywords: Beliefs; Game; Teachers; Experience and context.

\footnotetext{
Artículo resultado del proyecto de investigación realizado para optar el título de Magíster en Educación de la Universidad de La Salle, Bogotá, 2015.

2 Magíster en Docencia. Universidad de la Salle. E-mail: contacto@diana.com.co

${ }^{3}$ Magister en Educación. Profesora de la Fundación Universitaria del Área Andina: angelescorp1@yahoo.com y Profesora de la Fundación Universitaria Monserrate Unimonse-rrate: johannasanchezmd@gmail.com
} 


\section{Introducción}

Este artículo da cuenta de la investigación realizada en el marco del Macro proyecto titulado "Sujetos y objetos de mediaciones pedagógicas: juego y educación", de la Universidad de la Salle, en el programa de Maestría en Docencia, encaminado a aportar, desde la investigación, conocimientos que respondan a realidades presentes en el contexto educativo. Las investigadoras, dos licenciadas y docentes del Programa de Licenciatura en Educación Preescolar de la Fundación Universitaria Monserrate - Unimonserrate, se interesaron por indagar y comprender el juego en el contexto universitario $y$, aún más, en un programa que ha tenido una trayectoria en el estudio de la dimensión lúdica en los niños y niñas.

Resulta importante mencionar que el juego necesita ser visible con un sentido pedagógico $\mathrm{y}$ didáctico en todos los niveles educativos $\mathrm{y}$, en particular, en las prácticas pedagógicas de los docentes. Es allí donde surge el interés de las investigadoras por comprender el lugar del juego en la dinámica de los programas de formación de maestros, además porque esta es una de las apuestas más importantes en la educación inicial, como lo señala el Documento No. 22 de la Serie de orientaciones pedagógicas para la educación inicial en el marco de la política pública de atención integral a los niños:

El juego es una de las actividades rectoras de la infancia, junto con el arte, la literatura y la exploración del medio ya que se trata de actividades que sustentan la acción pedagógica en educación inicial y potencian el desarrollo integral de las niñas y los niños, también deben estar presentes en acciones conjuntas con la familia a través de la valoración e incorporación de su tradición lúdica, representada en juegos, juguetes y rondas. (p. 18)
Siendo esta una orientación para la educación inicial en nuestro país se espera que, en la formación de maestros para este nivel, el tema del juego esté presente en los procesos de enseñanza y de aprendizaje, de manera que posteriormente se proyecte en el trabajo con los niños y las niñas.

A través de la historia el juego ha sido estudiado para reconocer su papel en la cultura y en la educación; diferentes teorías han considerado el juego como constitutivo del ser humano, promotor y creador de la subjetividad básica para concretar y estimular el aprendizaje; desde el campo educativo es importante reconocer la mediación pedagógica del juego y su relación con la formación de la primera infancia, pues es a través de él que los niños acceden al conocimiento del mundo que los rodea.

Por ejemplo, Johan Huizinga (1972) afirma que la cultura surge en forma de juego y que esta se desarrolla a partir de él; establece también que el juego posee una función humana tan esencial como la reflexión y el trabajo; el Homo faber, u "hombre que trabaja", y el Homo ludens, u "hombre que juega", se complementan. Conforme a este planteamiento, el entorno educativo debe articular el juego desde su función social y cultural, pues además de ser constitutivo del sujeto, concreta y estimula el aprendizaje de nuevas experiencias de lenguaje y de cognición.

No obstante, en el contexto educativo el juego ha sido considerado, muy frecuentemente, como pérdida de tiempo y se observa cómo sigue ausente en los diferentes niveles del desarrollo o, del sistema educativo); por ejemplo en la formación de adolescentes y se cuenta con un mínimo de actividades lúdicas para favorecer los procesos de aprendizaje; las clases magistrales y las didácticas tradicionales siguen marcando la pauta dentro de los espacios de enseñanza, quizás por desconocer las posibilidades del juego en relación con el aprendizaje. 
Es así como el interés por comprender las creencias de los docentes acerca del juego, en los programas de formación de maestros para la educación inicial, llevó a las investigadoras a plantear esta investigación en torno a la categoría de creencias, por considerar que ellas guardan un vínculo directo con la experiencia histórica, social y cultural de todo ser humano y porque fundamentan y orientan sus acciones en la interacción con otros.

En el marco de este proyecto de investigación, se entiende la creencia desde los planteamientos de Luis Villoro (1982), quien la concibe como un estado disposicional adquirido en el sujeto, que causa un conjunto coherente de respuestas y que está determinado por un objeto o situación aprendida, y que dicho estado orienta la práctica del sujeto ante el mundo.

En la búsqueda de construcción de conocimiento que aporte a la comprensión del juego como mediación pedagógica, se planteó la siguiente pregunta de investigación ¿Cuáles son las creencias sobre el juego de docentes en dos programas de formación de maestros para la educación inicial?

\section{Metodología}

Esta investigación se realizó desde el enfoque metodológico cualitativo, siguiendo las orientaciones de Tezanos (1998) quien plantea que las circunstancias o caracteres, naturales o adquiridos, que distinguen a las personas y a las cosas, proporcionan explicaciones e interpretaciones de su actuar en las interacciones con el medio y con los otros. Igualmente, la investigación retoma los aportes de Torres (1996), quien asume que, de modo abierto y flexible, la investigación cualitativa emplea diversas herramientas e instrumentos para su desarrollo, aplicabilidad y análisis, permitiendo la interpretación, a profundidad, de los hechos que se investigan, más allá de los datos estadísticos que puedan obte- nerse de ellos.

Se tomó en cuenta la perspectiva epistemológica hermenéutica, planteada por Álvarez (2003); se trabajó desde la voz de los participantes elegidos en diferentes contextos: un grupo de docentes de dos programas de formación para maestros de la educación inicial en Bogotá, y desde la comprensión e interpretación de sus creencias, en relación con el lugar del juego en los procesos de formación en educación superior. Se trató de un estudio exploratorio, ya que no se conocen estudios previos al respecto.

Se estableció como criterio de selección de las instituciones que una de ellas contemplara el juego dentro de la malla curricular y la otra no. En este orden de ideas, las dos instituciones seleccionadas fueron: la Fundación Universitaria Monserrate -Unimonserrate y la Pontificia Universidad Javeriana. Se investigaron las creencias acerca del juego en un grupo conformado por 5 docentes de cada institución, para un total de 10, con las siguientes características:

1. De ambos géneros.

2. Con experiencia docente en el programa de 1 a 3, 5 a 8 y de 10 o más años.

La técnica de recolección de la información fue de corte cualitativo, a través de la herramienta denominada entrevista semi-estructurada, individual, sobre creencias acerca del juego como mediación pedagógica en la práctica personal de cada docente. Según Hernández et al (2010) se entiende la entrevista como un instrumento que apunta a obtener respuestas sobre un tema o problema desde el lenguaje y perspectiva del entrevistado, en un espacio que favorece la naturalidad, la espontaneidad y la amplitud de respuestas.

Para el análisis del contenido de las voces recogidas durante las entrevistas se tomaron en cuenta los planteamientos de Torres (1996), quien 
propone cuatro niveles de análisis:

- Categorización y codificación

- Ordenación y clasificación

- Establecimiento de relaciones

- Establecimiento de redes de sentido

categorización y codificación: se agrupó y se ordenó la información estableciendo un modo de lectura que facilitara el manejo de los hallazgos; ubicando temas agrupadores de información, buscando pasar de una mirada global de la realidad a una más estructurada y lógica del objeto de estudio. Para la codificación se asignaron códigos que facilitaron el manejo de la información, establecidos por números, letras u otros símbolos, ejemplo E.1.F.U.M.C.C Entrevista 1, Fundación Universitaria Monserrate: Carlos Castañeda

Posteriormente se procedió a la ordenación y clasificación, se relacionaron las preguntas establecidas al interior del guion de la entrevista semiestructurada con la información dada por los participantes, iniciando el establecimiento de recurrencias dentro de ella y algunas observaciones bajo la lectura de las investigadoras. De las recurrencias se derivaron las categorías iniciales y emergentes dando paso al establecimiento de relaciones donde a través de la lectura y organización de la información se propuso una ordenación estructural a través de relaciones del comportamiento del objeto de estudio: creencias sobre el juego. Finalmente, en el cuarto nivel de análisis el establecimiento de redes de sentido, se pusieron en diálogo las categorías iniciales y las categorías emergentes, en la búsqueda de profundizar en cada uno de los hallazgos encontrados desde la voz de los actores claves.

Finalmente, se hizo un análisis de los planes de estudio de las dos instituciones donde laboraban los docentes, contrastando como cada institución hace una apuesta diferente frente al juego dentro de sus prácticas de formación.

\section{Resultados y discusión}

Este apartado se desarrollará en dos partes. La primera, orientada a las categorías emergentes, entendidas como las creencias identificadas a partir de la información obtenida a través de las entrevistas. La segunda dará cuenta de las relaciones establecidas entre las creencias, a partir del establecimiento de redes de sentido.

\section{Creencias identificadas sobre juego}

Partiendo de las dos grandes categorías deductivas iniciales de la investigación, creencias sobre el juego y didáctica, el proceso metodológico inductivo permitió establecer ocho categorías emergentes, entendiendo emergente como aquello que se descubre en la lógica de la lectura:

- Noción de juego

- Uso del juego

- Tipos de juego

- Juego en la formación de maestros

- Lúdica como dimensión

- Didáctica en la educación superior

- Juego y didáctica

- Metas y estrategias didácticas

Estas categorías fueron además registradas como las creencias que identifican modos de ser de los docentes que participaron en la investigación. En este sentido es importante retomar la conceptualización de la creencia con los aportes de Villoro (1982), quien la define como un estado disposicional del ser con el cual se acerca a la comprensión y explicación de las situaciones o experiencias, que orienta la acción del sujeto ante el mundo y guarda siempre una relación estrecha con el querer y el interés.

La siguiente figura relaciona las ocho categorías de creencias, con un nombre que enuncia de manera general su contenido y una aproximación al significado que se desarrolla a continuación. 


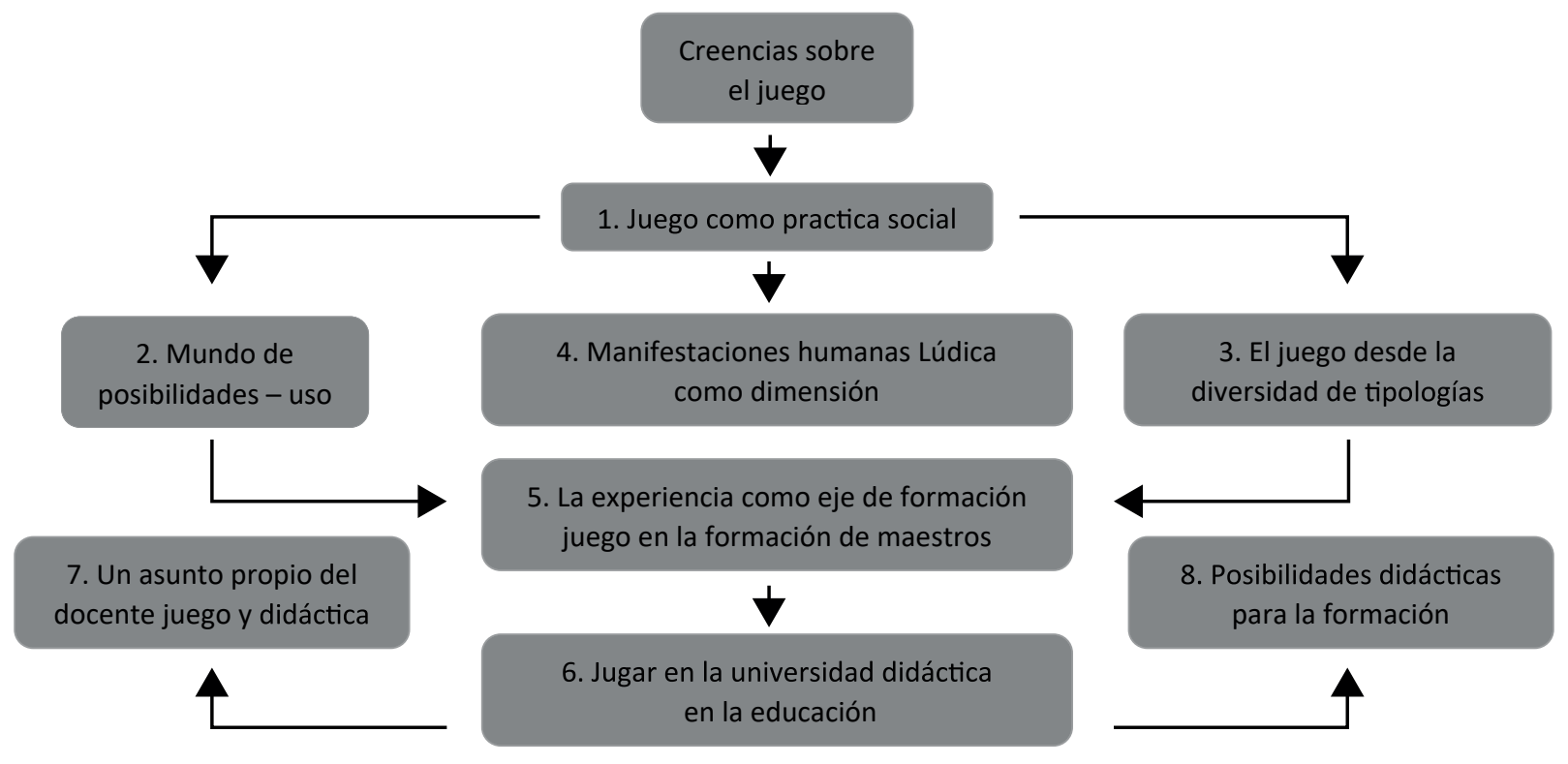

Figura 1. Categorías acerca de las creencias de los docentes sobre el juego y la didáctica Fuente: Elaboración propia

En la primera categoría emergente, el juego como práctica social, el juego se revela como una actividad innata en el ser humano que se construye como una práctica social, conformada por diferentes actividades que posibilitan el desarrollo y el aprendizaje en diferentes órdenes de la vida.

Esta creencia sobre el juego se aproxima a los planteamientos de Huizinga (1972) cuando se refiere al juego resaltándolo como una forma de construcción de la cultura propia del ser humano:

Complemento, parte de la vida misma en general (...) imprescindible para la persona, como función biológica y para la comunidad, por el sentido que encierra, por su significación, por su valor expresivo y por las conexiones espirituales y sociales que crea; en una palabra, como función cultural. (p. 21)

Segunda categoría emergente, Mundo de posibilidades: aquí el juego se comprende como posibilitador de experiencias y a su vez como la oportunidad para la relación consigo mismo y con los demás. Igualmente, en esta categoría se eviden- cian dos tensiones denominadas ventajas y desventajas, que revelan la posibilidad del uso u omisión del juego, desde la creencia acerca del juego en los programas de formación de maestros en pregrado y su influencia en las mismas instituciones donde se ejerce la labor docente.

Esta tensión aparece en la medida en que se logra identificar que existe una claridad sobre el uso del juego, entendido como una posibilidad, muy relacionada con la postura de Winnicott (1972), quien argumenta que sólo a través del juego el niño y el adulto ponen de manifiesto su capacidad creadora y es partir de él como el sujeto pone en juego su personalidad, y se descubre y se reconoce. También encontramos que cuando se establece la relación entre las ventajas y desventajas del uso del juego, este puede llegar a ser o no una acción significativa para el sujeto. Es decir, desde la creencia sobre el juego y la libre elección dentro de la experiencia del sujeto, puede determinarse si se hace uso o no en el desarrollo de la práctica docente.

Tiene muchas ventajas en la formación en todo lo que tiene que ver con la dimensión 
socio-afectiva y con todo el desarrollo que tiene que ver con la moral y los juicios. Por ejemplo, he encontrado que es interesante el juego para descentrar a las estudiantes. (E.4.F.U.M.M.B)

El juego indudablemente en el terreno de pensamiento es la posibilidad significativa de ser, sentir y pensar de otras maneras. (E.8.P.U.J.J.Z)

Una desventaja sería caer en el activismo si no se tiene clara la reflexión de lo que se hace. (E.1.F.U.M.C.C)

Es necesario que el juego esté acompañado de una reflexión que supere el placer lúdico y que se piense en un conocimiento disciplinar, en un saber pedagógico (E.5.F.U.M.N.H)

Tercera categoría emergente, el juego desde la diversidad de tipologías: los tipos de juego han sido establecidos desde las diversas disciplinas trabajadas por los docentes en el ejercicio de orientación y/o formación con maestros para la educación inicial; es decir, lo que el docente propone como apuesta de trabajo en el desarrollo de diferentes asignaturas como parte cercana al juego y a su posible clasificación; tales como lenguaje, artes, ciencias naturales, epistemología, comunicación oral escrita y literatura infantil, entre otras. Vale la pena señalar que, en su ejercicio docente, cada uno logra establecer una relación con elementos del juego que se ajustan a las temáticas desarrolladas. Sin embargo, no es clara una construcción conceptual de las tipologías de juego, lo que pone en evidencia que los participantes, tienen sus propias interpretaciones sobre dicha conceptualización.

Yo manejo muchos juegos que tienen que ver con la dimensión corporal y comunicativa, pues pienso que el juego me permite desarrollar su ser y en este caso sería con las maestras en formación de una manera integral. (E.2.F.U.M.L.P)
Juegos de lenguaje que acercan al estudiante al desarrollo de mi disciplina. (E.5.F.U.M.N.H)

Depende de la intencionalidad y del espacio formativo. A veces integro algunos juegos como, por ejemplo, juego de roles, juegos reglados, juegos de construcción colectiva y de lenguaje, pues son como lo más adecuados en la relación con mis temas. (E.10.P.U.J.C.R).

Cuarta categoría emergente, manifestaciones humanas: el juego está directamente relacionado con la lúdica, pues ella se orienta hacia manifestaciones como el goce, el placer y el disfrute, experimentadas en diferentes situaciones de la vida. El juego posee un carácter subjetivo, pues lo que es lúdico para unos no lo es para otros. Sin embargo, el juego sí logra establecerse como lúdico para el ser humano en general, pues el hecho de jugar lo coloca en otro contexto y le permite evidenciar dichas manifestaciones.

Establecer una relación entre lúdica y juego suele entrañar múltiples significados, en este sentido el grupo de docentes, logra evidenciar que existe la creencia de que la mayoría de las manifestaciones humanas que poseen características de goce, placer y diversión están dentro de la lúdica y por ende guardan una relación directa con el juego, siendo entonces como un punto de interacción consigo mismo, con los demás y con el mundo.

Quinta categoría emergente, la experiencia como eje de formación: el grupo de docentes coincide en que la formación de los maestros para la educación inicial tiene como eje de articulación la teoría y la práctica, siendo fundamental la experiencia como el elemento vital de engranaje de estos dos componentes; esto permite un ejercicio reflexivo y de transformación dentro de su rol de maestros, permitiéndoles avanzar en la construcción de su rol como profesionales.

Resulta importante afirmar que la práctica es la clave de la formación en la medida en que 
les permite a los maestros en formación asumir posturas frente a un objeto de estudio específico. Es así como se refleja la creencia de que la experiencia será posteriormente el insumo de su acción frente a procesos de enseñanza-aprendizaje. Es decir, si el maestro en formación logra vivenciar el juego dentro los espacios de clases a las que asiste, podrá hacerlo extensivo en su quehacer profesional con los niños y las niñas.

Sexta categoría emergente, jugar en la universidad: dentro del ejercicio investigativo se aprecia un contraste entre los dos grupos de las instituciones seleccionadas en este proyecto. Se vislumbra, por un lado, la creencia de que, dentro de los procesos de formación en el ámbito universitario, las didácticas son rigurosas y de carácter formal y que, a mayor nivel educativo, mayor presencia de otros modos de construcción de conocimiento, el juego no es uno de estos modos. En contraste, se observa la creencia de que el juego posee un valor importante y de significación dentro del rol de maestros para la educación inicial y se instaura como un mediador y posibilitador de desarrollo y construcción de saberes. Aunque se da este paralelo, las dos instituciones coinciden en la creencia de que no es una didáctica propia de la educación superior.

Entonces se evidencia que la didáctica y su articulación con el juego en la formación universitaria están distanciadas a partir de las creencias de los docentes, quienes ponen de manifiesto que la universidad es un espacio de rigurosidad académica, elemento que se desdibuja en el juego, o, igual de problemático, es concebido como una estrategia para la construcción de conocimiento. Por el contrario, Huizinga (1972) se refiere a la seriedad del juego como una acción que se transforma y que puede variar:
El valor inferior del juego encuentra su límite en el valor superior de lo serio, el juego se cambia en cosa seria y lo serio en juego. Puede elevarse a alturas de belleza y santidad que quedan muy por encima de lo serio. (p. 27)

Es decir, el juego no puede limitarse a algo superficial, ya que su seriedad radica en el valor que el mismo posee, al ser propio del sujeto.

Séptima categoría emergente, un asunto propio del docente: esta categoría se refiere al ejercicio docente en el que la reflexión, la formalización, los procesos y las posibilidades dadas antes, durante y después de las prácticas de enseñanza se convierten en tareas primarias de su quehacer. Tal ejercicio está inmerso en la didáctica como disciplina que toma los procesos mencionados y permite transformar próximas intervenciones.

De acuerdo con lo anterior, la didáctica forma parte fundamental del ejercicio docente y es en ella donde se articulan las creencias con las acciones y las intencionalidades. Es decir, el sujeto está constituido por sus creencias y hace o no réplica de ellas en su cotidianidad. Además, estas pueden ser modificadas o transformadas por variables propias del entorno. Es por esta razón que el juego no se hace evidente en sus prácticas, aunque poseen ciertas creencias sobre el juego no las tienen en cuenta a la hora de planear y organizar sus clases, por ejemplo, porque en la institución donde laboran no tiene referenciado el juego como mediación pedagógica.

Octava Categoría emergente, posibilidades didácticas para la formación universitaria: las didácticas del ámbito universitario y específicamente de los programas de formación de maestros para la educación inicial, son transversadas por la sumatoria de creencias constituidas desde la experiencia de cada docente, siendo este el punto de partida para identificar cuáles son los modos más 
asertivos y pertinentes en su acción, teniendo en cuenta la disciplina de la cual se trate. Algunas posibilidades didácticas retoman elementos y características propias del juego como vehículo facilitador dentro del proceso de aprendizaje, pero no son concebidas directamente como una didáctica.

Es necesario aclarar que estas categorías fueron analizadas a la luz de los contextos donde laboraban los docentes quienes hicieron parte de la investigación, Por un parte la Pontificia Universidad Javeriana, dentro de la formación de licenciadas en Pedagogía Infantil, maneja un plan de estudios que aleja el juego de manera implícita en la propuesta de formación. Algunos docentes afirmaron que la universidad en su momento tenía un énfasis más cercano a la línea de lenguaje.

Caso contrario ocurrió con la Fundación Universitaria Monserrate. Dentro del plan de estudios para la formación de licenciadas en Educación Preescolar, la Unimonserrate concebía la lúdica y la creatividad como un eje de formación permanente e importante. Ambas apuestas de formación se corroboraron al observar en el número de asignaturas que relacionaba cada institución con elementos propios sobre juego.

En este corto análisis de los planes de estudio se pudo comprender que las apuestas institucionales afectaron las creencias y las relaciones que establecieron los docentes en torno al juego. Los docentes transmiten diferentes creencias sobre el juego, pero al estar inmersos en las prácticas de los programas, se adaptan o flexibilizan su pensar y su actuar bajo las orientaciones dadas por el contexto institucional. Cada institución adopta una clara postura frente a sus propósitos formativos.

A manera de conclusión, cabe aclarar que las creencias encontradas en esta investigación sobre el juego están asociadas con la didáctica en la medida en que se presenta como un elemento que rompe la rigurosidad en el ámbito universitario, favoreciendo procesos de construcción de conocimiento.
Aunque el grupo entrevistado cree que el juego no es una didáctica, logran integrarlo dentro del desarrollo de sus encuentros de formación con los estudiantes. Vale la pena señalar que las dos últimas categorías tienen como eje principal la didáctica. Sin embargo, la séptima categoría corresponde a un concepto general y la octava a posibilidades específicas para el desarrollo de las diferentes asignaturas.

Este trabajo permitió identificar, agrupar y caracterizar un conjunto de creencias que circulan en el discurso y en la práctica de los docentes indagados en la investigación. Vale la pena indicar que el proceso de análisis y comprensión se distancia de señalar errores y equivocaciones en las creencias. Son más bien entendidas como insumo para comprender a fondo las motivaciones, los conocimientos, las experiencias que anteceden a las prácticas específicas. Esta información es vital para la propuesta de una red de sentido elaborada por las investigadoras a partir de las informaciones suministradas por los actores claves. Se trata entonces de evidenciar las relaciones entre las creencias sobre juego y la didáctica en el contexto de dos programas de formación de maestros para la educación inicial.

Aunque se trata de una red de sentido propuesta por las investigadoras, esta se deriva de la articulación entre las dos categorías iniciales, creencias sobre el juego y didáctica, y las categorías emergentes, -noción de juego, tipos de juego, juego en la formación de maestros, lúdica como dimensión didáctica en la educación superior, juego y didáctica, metas y estrategias didácticas.-Desde allí partimos para la construcción de posibles relaciones y articulaciones que permitieran dar sentido a la información hallada.

\section{Identificación de posibles relaciones}

Partiendo del desarrollo de las categorías emergentes, se explicitan aquellas relaciones establecidas durante el proceso de la investigación. Se proponen varios tipos de relaciones comprendidas 
por las investigadoras como semejantes, contradictorias y flexibles, de la siguiente manera:

- Relaciones semejantes, aquellas que poseen elementos similares que tejen un sentido en torno a un objeto en común.

- Relaciones contradictorias, aquellas que entran en tensión por poseer diferencias dentro del mismo objeto de estudio.

- Relaciones flexibles, aquellas que se adaptan a las variables dadas en el contexto.

Otro tipo de relaciones son las que se dan entre la noción de juego y la acción del docente es a partir de las creencias sobre el juego donde se empiezan a tejer las relaciones. El juego como objeto de estudio se ha identificado desde una construcción polisémica constituida por diferentes orígenes de la creencia. Aquí es indispensable aclarar que sólo se hará hincapié, en detalle, en el origen de la creencia en esta relación, por considerarlo base de las siguientes relaciones que subyacen en el análisis.

Ahora, siguiendo los planteamientos de Villoro (1982), se evidencian tres orígenes de las creencias: antecedentes, motivos y razones, han sido tomados en cuenta en la articulación con las evidencias de los docentes que participaron en la investigación.

- Primero: por antecedentes, aquellas que colocan a los sujetos en una posición de aprendizaje y son adquiridas a lo largo de la vida por diferentes experiencias que traen consigo una construcción de conocimiento. Existe una situación o hecho biográfico que trae consigo una respuesta a la comprensión de juego y que se configura como creencia cuando es retomada para responder al concepto. Además, el sujeto trae consigo su experiencia cuando se requiere de ella sobre algo en particular.
- Segundo: por motivos, todas aquellas intenciones, deseos y necesidades de un sujeto ante un fin determinado que lo movilizan a actuar de cierta manera.

- Tercero y última, por razones, aquellos argumentos que dan explicación lógica y racional a lo que se cree.

La anterior descripción de las formas de origen de la creencia muestra que cada docente tiene una manera particular de construir la creencia de acuerdo con sus vivencias, formación y condiciones del contexto; la diferencia entre las tres formas tiene que ver con la raíz de la creencia, antecedentes una situación, motivos un sentimiento y razones un argumento. Sin embargo, dichas creencias no determinan las acciones del sujeto.

Se concluye este análisis mencionado la importancia de conocer la relación que los docentes proponen entre juego y didáctica a través del reconocimiento y caracterización de las creencias, entendiendo que los maestros que participaron en la investigación han elaborado a través de su experiencia una cierta inconsistencia entre la creencia y la práctica. Existen muchos elementos teóricos que fundamentan el juego, pero pocas prácticas que lo repliquen. Se torna entonces necesario hacer una articulación con estos elementos básicos para que a partir de allí se dé una resignificación del juego como acción de construcción social y de aprendizaje

\section{Conclusiones}

Comprender las creencias de algunos de los docentes de programas de formación de maestros para la educación inicial ha sido una oportunidad para construir un horizonte de sentido frente a las maneras como se configuran algunas prácticas relacionadas con el juego dentro de estos espacios de formación. Identificar las creencias del grupo de docen- 
tes entrevistados permitió los primeros acercamientos al pensamiento docente respecto del juego, siendo este un primer paso para la caracterización de las mismas y para determinar las relaciones que se establecen a partir de su creencia y su hacer en el contexto educativo.

Como se observa en la Figura No 2, la creencia sobre el juego fue el núcleo de la investigación y alrededor de ella se dieron los diferentes hallazgos; es pertinente aclarar que la organización que se presenta no se da de manera jerárquica, pues la investigación mostró que las creencias se relacionan todo el tiempo. Si bien existe una importancia en ellas, no se pueden describir como unas más importantes que otras.

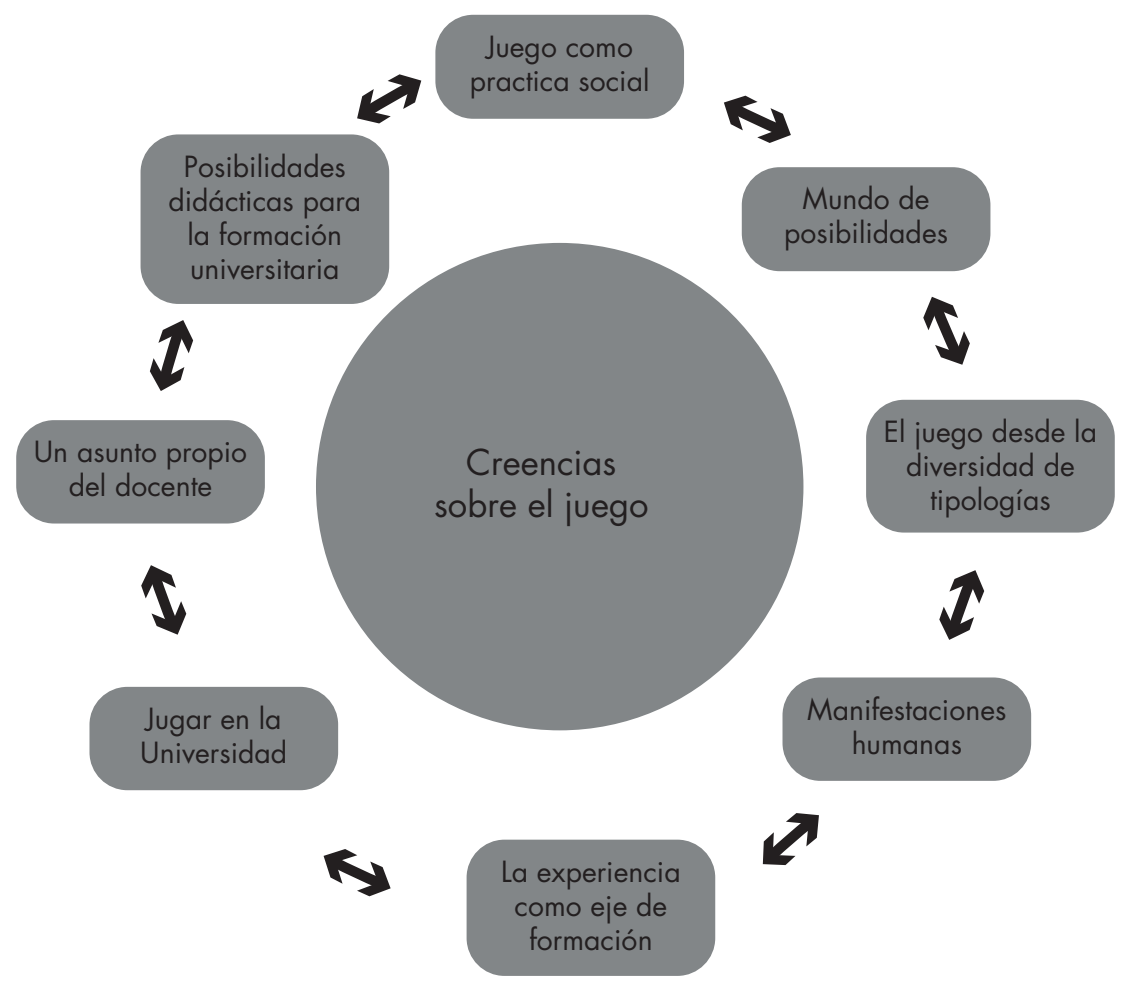

Figura 2. Las creencias como sistema.

Fuente: Elaboración propia

Después de analizar las creencias sobre el juego, se plantearon seis conclusiones:

- Primera, las creencias de los docentes participes de la investigación están mediadas por las experiencias y la influencia del contexto en el que se desenvuelven, elementos que constituyen en la base fundamental.

- Segunda, para los participantes el juego es de vital importancia en el ser humano y de manera especial para los maestros en formación de los programas para la educación inicial, pues se convierte en un referente básico para su ejercicio profesional y un posibilitador de mediaciones pedagógicas que acompañen el desarrollo de la persona desde la infancia.

- Tercera, el juego no es una didáctica en los programas de formación donde laboran los docentes, se establece como una mediación pedagógica. Por cumplir una función social y cultural, el juego no puede ser instrumentalizado, sino utilizado desde su propósito en sí mismo.

- Cuarta, se puede destacar la relación existente entre la creencia y la práctica. La investigación 
corrobora lo planteado por Villoro en lo concerniente a que la creencia no siempre se configura en un elemento vital en el desarrollo de la práctica.

- Quinta, desde el escenario investigativo, existe en los docentes un estigma por el juego desde sus creencias acerca del mismo como algo lejano de esta etapa educativa. Solo se hacen conscientes de él en ámbitos propios de su relación con los niños y las niñas.

- Sexta y última: salta a la vista la dificultad que reviste, en la comprensión de la dimensión del juego desde sus características intrínsecas, pues suelen ser ignoradas en los procesos de enseñanza y de aprendizaje.

La investigación se propuso hacer un aporte en relación con el juego en los programas de formación de maestros para la educación inicial, desde el horizonte de sentido del juego como posibilitador de la construcción social y gestor de la cultura; constituye una invitación a evocar nuevamente el juego desde su valor social; a descubrirnos desde la espontaneidad, el goce y el disfrute de un instante de juego, que nos saque de la rutina y nos muestre el valor de lo sencillo. Es la oportunidad de retomar este elemento fundamental de desarrollo personal, académico, profesional y cultural

Se espera que los resultados de esta investigación sean una provocación a asumir el juego desde el ámbito universitario, en los procesos de formación de maestros para la educación inicial, aportando de esta manera a la configuración y aplicación de política pública en relación con la atención y formación integral de los niños y niñas de este país.

\section{Referencias}

Álvarez, J. y Godoy J. (2003). Cómo hacer investigación cualitativa. Fundamentos y metodología. México: Paidós.

Hernández, R., Fernández, C., y Baptista, P. (2010). Metodología de la investigación. México: McGraw-Hill Interamericana Editores.

Huizinga, J. (1972). Homo ludens. Madrid: Emecé Editores.

Ministerio de Educación Nacional de Colombia MEN (2012). El juego en educación inicial. Documento 22 de la serie de orientaciones pedagógicas para la educación inicial en el marco de la atención integral. Recuperado de:

http://www.deceroasiempre.gov.co/Prensa/CDocumentacionDocs/Documento-N22-juego-educacion-inicial.pdf

Tezanos, A. (1998). Una etnografía de la etnografía. Aproximaciones metodológicas para la enseñanza del enfoque cualitativo-interpretativo para la investigación social. Bogotá: Antropos Ediciones.

Torres, A. (1996). Estrategias y técnicas de investigación cualitativa. Colombia: Editora Guadalupe.

Villoro, L. (1982). Creer, saber y conocer. México: Siglo XXI Editores.

Winicott, D. (1972). Realidad y juego. Barcelona: Gedisa. 